\title{
Interpreting measurements of cardiac function using vendor- independent speckle tracking echocardiography in children: a prospective, blinded comparison with catheter-derived measurements
}

\author{
Suma P. Goudar, M.D. ${ }^{1}$, G. Hamilton Baker, M.D. ${ }^{2}$, Shahryar M. Chowdhury, M.D., M.S.C.R. ${ }^{2}$, \\ Kimberly J. Reid, M.S. ${ }^{1}$, Girish Shirali, M.B.B.S. ${ }^{1}$, and Mark A. Scheurer, M.D., M.S.C.R. ${ }^{2}$ \\ ${ }^{1}$ Ward Family Heart Center, Children's Mercy Hospital, Kansas City, Missouri \\ ${ }^{2}$ Department of Pediatrics, Division of Pediatric Cardiology, Medical University of South Carolina, \\ Charleston, South Carolina
}

\section{Abstract}

Background-Adult studies demonstrate that echocardiographic measurements of cardiac function using speckle tracking correlate with invasive measurements, but such data in the pediatric population are sparse. Our aim was to compare speckle-derived measures of cardiac function to measurements routinely obtained by cardiac catheterization in children.

Methods-Echocardiograms were performed on the day of cardiac catheterization. Using Tomtec 2D Cardiac Performance Analysis, longitudinal strain (LS), longitudinal strain rate (LSR), early diastolic LSR, and ejection fraction (EF) for the right and left ventricle (RV and LV) were calculated via speckle tracking. Global LS and LSR were calculated for the LV. These results were compared to cardiac index, maximum ventricular $\mathrm{dp} / \mathrm{dt}(\max \mathrm{dp} / \mathrm{dt}$ ), ventricular end-diastolic pressure (EDP), and pulmonary capillary wedge pressure (PCWP) obtained by fluid-filled catheters. A blinded observer performed all echo measurements.

Results-Fifty studies were performed on 28 patients ages 4 months to 20 years old. Their diagnoses included cardiac transplant (48 studies), repaired AV septal defect (1), and dilated cardiomyopathy (1). RVEDP ranged from 2 to $22 \mathrm{~mm} \mathrm{Hg}$ (median=6) and PCWP ranged from 6 to $32 \mathrm{mmHg}$ (median 10). LV global LS and LV 2-chamber LSR by speckle-tracking negatively correlated with LV max dp/dt (LV global LS $R=-.83, P=.001$; LV 2-chamber LSR $R=-.69, P=$. $009)$. RV LS weakly correlated with max $\mathrm{dp} / \mathrm{dt}(R=.363, P=.002)$. Early diastolic strain rate did not correlate with EDP in either ventricle.

Conclusion-Speckle-derived measurements of function in the LV have stronger correlation than the RV to catheter-derived measures. LV global LS has the strongest correlation with invasive function measures in children.

Correspondence Suma P Goudar, Ward Family Heart Center, Children's Mercy Hospital, Kansas City, MO, USA. spgoudar@cmh.edu. 


\section{Keywords}

cardiac imaging; diastolic function; myocardial strain; systolic function

\section{INTRODUCTION}

Close monitoring of the cardiac function of pediatric patients with heart disease is critical in longitudinal follow-up. The gold standards for evaluating cardiac function in children are cardiac catheterization and MRI. Both of these modalities are costly and often require general anesthesia for children, which carries a significant risk, particularly in patients with underlying heart disease. Cardiac catheterization in addition involves the use of radiation and is an invasive procedure. Echocardiography is a portable and noninvasive modality that is well suited for serial study.

Currently used quantitative methods of measuring cardiac function by echocardiography have important limitations in children. In a study of 20 children comparing M-mode, twodimensional, and three-dimensional echocardiographic measures of left ventricular function to MRI ejection fraction, three-dimensional ejection fraction had the strongest correlation to MRI and the lowest inter- and intra-observer variability. ${ }^{1}$ Ejection fraction is loaddependent, however, and 3D ejection fraction is time-consuming to calculate and often requires breath-hold and stillness during the acquisition for accuracy, which is difficult in children. The ability to evaluate right ventricular function is more challenging due to the complex geometry of this ventricle.

Speckle tracking is a promising new technique to measure cardiac function by echocardiography. The technology tracks the motion of speckles (natural acoustic reflections and interference patterns within user-defined regions of interest), which determines tissue motion and deformation. From this, strain and strain rate of the myocardium can be calculated and are presumed to be measures of myocardial function. Speckle tracking echocardiography is angle-independent and regarded as less load-dependent than ejection fraction. From a practical standpoint, echocardiographic images can be rapidly acquired at the bedside and can either be analyzed off line or on line. Speckle-derived strain and strain rate of the left ventricle correlate well to systolic strain by tagged MRI and sonomicrometry in human and animal models. ${ }^{2-4}$ In adults with repaired tetralogy of Fallot, ventricular global longitudinal strain correlates well with MRI EF. ${ }^{5}$ Furthermore, prior adult studies demonstrate that speckle-derived strain and strain rate measurements correlate with left ventricular end-diastolic pressure. ${ }^{6-8}$

In the pediatric population, studies show that longitudinal strain is more reproducible than circumferential and radial strain. ${ }^{4,9}$ Normal values of left ventricular strain ${ }^{10,11}$ and right ventricular strain ${ }^{12}$ have been published. There are limited studies in the pediatric population comparing speckle-derived measures of cardiac function to catheter-derived measures of cardiac function to date, and these studies primarily evaluate right ventricular strain in subjects with either pulmonary hypertension or single ventricle anatomy. ${ }^{13-15}$ The aim of this study was to compare speckle-derived measurements of cardiac function to 
catheter-derived measures of cardiac function by cardiac catheterization in children with biventricular hearts and a systemic left ventricle.

\section{METHODS}

\subsection{Study population}

The study group consisted of pediatric patients with biventricular hearts and a systemic left ventricle who were undergoing cardiac catheterization with thermodilution cardiac outputs performed for clinical indications at our institution between May 2011 and April 2012. All subjects who had cardiac catheterization and echocardiogram ordered on the same day were considered for the study. Exclusion criteria included single ventricle anatomy, systemic right ventricular anatomy, and shunt lesions (such as PDA or ASD). Eligible subjects were enrolled consecutively. The institutional review board approved this study without consent of subjects as the studies were performed for clinical purposes as standard of care at our institution and data recorded was de-identified of personal information.

\subsection{Study protocol}

2.2.1 Cardiac catheterization and invasive hemodynamics-Patients had sedation or general anesthesia during the cardiac catheterization as clinically indicated by the anesthesia team. All hemodynamic measurements were performed using a fluid-filled thermodilution catheter ( 5 or 7 French). Pressures were recorded after heparinized saline catheter flush and inspection of the waveform to assure appropriate catheter position and recording. Hemodynamic measurements were recorded prior to angiography or any indicated interventional procedure. The cardiac index and cardiac output were calculated during the procedure and recorded by the physician performing the catheterization. Standard thermodilution methods were used with saline injections. The average of three measurements was used for the final calculation of cardiac output and cardiac index. Philips Xper software was used for waveform analysis, identification of end-diastolic pressure, and calculation of max dp/dt by a single, blinded observer (SG). This software interpolates pressure measurements at $250 \mathrm{~Hz}$ from the unfiltered catheter data. The max dp/dt was calculated as the maximum value of the derivative of pressure and time and averaged over 10 cardiac cycles.

2.2.2 Echocardiographic technique-All echocardiograms were performed on the same day of cardiac catheterization, but not simultaneously while invasive catheter measurements were performed. A large majority of echocardiograms was performed, while patients were still under the same general anesthesia. The echocardiograms were performed in a supine and left lateral decubitus position. The echocardiograms were performed from the left side using the Phillips iE33 system (Andover, MA, USA). Apical four-chamber views of the right and left ventricle, and apical two-chamber views of the left ventricle were recorded in three beat loops. All images were sent to a research server in DICOM format compressed to 30 frames/s for offline analysis.

2.2.3 Speckle tracking analysis-A single, blinded observer (SG) performed offline analysis of DICOM images using vendor-independent software (Tomtec 2D Cardiac 
Performance Analysis). This analysis was performed at a separate time point from the cardiac catheterization pressure analysis (at least 3 weeks) to minimize recollection bias. Points of interest were placed in the mid-myocardium from the level of the atrioventricular valve annulus to the apex at a single point in the cardiac cycle. The resulting tracing was visually inspected throughout the cardiac cycle to determine adequate tracking of the myocardium. The points of interest were adjusted as necessary until the tracing visually appeared to track the myocardium adequately. A second observer (GS) who was blinded to the catheter-based measurements and patient name reviewed all tracings to verify the echocardiographic measurements. Images were graded for quality of tracking by both observers:

1. Grade 1-all segments tracked well

2. Grade 2-one-two segments tracked poorly but remainder tracked well

3. Grade 3-more than two segments tracked poorly.

Speckle tracking analysis on grade 1 and grade 2 images was performed to calculate peak longitudinal strain, peak longitudinal strain rate, peak early diastolic longitudinal strain rate and ejection fraction in the apical view for the right ventricle and in the apical four- and twochamber views for the left ventricle. Figure 1 depicts images from the software analysis program and how the measurements were performed. Right ventricular longitudinal strain was calculated as an average of six segments (three lateral and three septal). The four- and two-chamber values were averaged to derive global left ventricular peak longitudinal strain, global peak left ventricular longitudinal strain rate, and global peak early diastolic longitudinal strain rate. The software uses single plane Simpson's method from the speckle tracking analysis to calculate ejection fraction for each view of both ventricles.

A third blinded observer (YC) performed echocardiographic analysis using speckle tracking on 10 studies for inter-observer reproducibility.

\subsection{Statistical analysis}

Continuous variables were not normally distributed and therefore are presented as median (range). Spearman's correlation coefficients were used to assess associations between speckle tracking variables and hemodynamic measurements at catheterization. Intra-class correlation coefficient (ICC) was performed for inter-observer reproducibility of echocardiographic measurements. An alpha level <.05 was considered statistically significant. Analyses were performed with SPSS version 20.0 (SPSS Inc, Chicago, IL, USA).

\section{RESULTS}

\subsection{Patient population}

A total of 50 studies were performed on 28 subjects. The subjects ranged from 4 months to 20 years of age (median 4 years). Their weight ranged from 6 to $72.8 \mathrm{~kg}$ (median $15.7 \mathrm{~kg}$ ) and body surface area from 0.28 to $1.88 \mathrm{~m}^{2}$ (median 0.64). Patient diagnoses included heart transplant (27 patients), repaired AV canal defect, and dilated cardiomyopathy (one patient 
each). Two of the subjects who had a heart transplant had rejection. In all 50 studies, a right heart catheterization and echocardiogram was performed. Sixteen of the 28 subjects also had a single left heart catheterization; left ventricular end-diastolic pressure and left ventricular $\mathrm{dp} / \mathrm{dt}$ was measured in this subset of subjects. Of the 28 subjects, 20 had a single right heart catheterization, three had two right heart catheterizations, one patient had three right heart catheterizations, one patient had four right heart catheterizations, one patient had five right heart catheterizations, and two patients had six right heart catheterizations. No subject had more than one left heart catheterization. Overall, a total of 50 right heart measurements in 28 subjects and 16 left heart measurements in 16 subjects were incorporated into the correlation analysis between echocardiographic and catheterization data. See Table 1 for ranges of values for end-diastolic pressure, pulmonary capillary wedge pressure, max dp/dt, strain, strain rate, and speckle-derived ejection fraction.

\subsection{Image quality}

See Table 2 for image quality data for the images analyzed. For the right ventricle, $12 \%$ of images were given a grade 3 for quality and were unable to be analyzed. For the fourchamber view and two-chamber view of the left ventricle, $6 \%$ and $12.5 \%$, respectively, were given a grade of 3 for quality and were rejected for analysis.

\subsection{Systolic parameters}

Right ventricular max dp/dt ranged from 99 to $748 \mathrm{mmHg} / \mathrm{s}$ and cardiac index from 1.84 to 4.62 L/min $/ \mathrm{m}^{2}$. Left ventricular max dp/dt ranged from 726 to $2127 \mathrm{mmHg} / \mathrm{s}$ (Table 1). Right ventricular longitudinal strain had a weak, positive correlation with max dp/dt (Table 3 ) and did not correlate significantly with cardiac index. Left ventricular global longitudinal strain correlated well with max dp/dt (Table 3), but longitudinal strain for isolated views of the apical four- and two-chamber views did not correlate with max dp/dt. Longitudinal strain rate correlated with max dp/dt for the two-chamber left ventricle view (Table 3), but not in the apical four-chamber view. Correlation plots between left ventricular max dp/dt and LV two-chamber strain rate and left ventricular global longitudinal strain are illustrated in Fig. 2. Right ventricular longitudinal strain rate and speckle-derived ejection fraction for both ventricles did not correlate with cardiac index or max dp/dt. When only including grade 1 (highest quality) images, there was no improvement in the correlation of longitudinal strain with max dp/dt for the right ventricle, but there was a slight improvement in correlation of longitudinal strain with max dp/dt for the left ventricle (Table 4). None of the specklederived echocardiographic parameters correlated with cardiac index. See Fig. 3 for sample images of catheter pressure tracing and speckle-derived strain and strain rate analysis of study subjects with normal and poor ventricular function.

\subsection{Diastolic parameters}

Early diastolic strain rate did not correlate with end-diastolic pressure for either ventricle (Table 3), even when only grade 1 images (highest quality) images were analyzed (Table 4). Interestingly, left ventricular longitudinal strain rate of the two-chambered view, a systolic echocardiographic parameter, correlated with end-diastolic pressure (Table 3). 


\subsection{Reproducibility}

Inter-observer reproducibility of the echocardiographic measurements of longitudinal strain, strain rate, and early diastolic strain rate is illustrated in Table 5. Overall, there is good interobserver reproducibility of longitudinal strain and longitudinal strain rate for the right and left ventricle and early diastolic strain rate for the right ventricle. Correlation between observers was moderate for early diastolic strain rate of the left ventricle.

\section{DISCUSSION}

Of the speckle-derived strain parameters evaluated in this study, global longitudinal strain of the left ventricle had the strongest correlation with max dp/dt. Right ventricular longitudinal strain had no meaningful correlation with catheter-derived measurements of cardiac function in this cohort. This is in concordance with other studies performed in the pediatric single ventricle population that showed poor correlation between invasive catheter-derived systolic function parameters and right ventricular longitudinal strain. ${ }^{13,14}$ This may be explained by the fact that multiple views are obtained for left ventricular global longitudinal strain and may be improved if more views of the right ventricle are obtained for analysis as one does for the left ventricle. In addition, the right ventricle is overall more challenging to image adequately (even in the apical long-axis view), as evidenced by the fact that a higher proportion of the right ventricle images was rejected from analysis in this study compared to the left ventricle four chamber (12\% vs $6 \%)$.

To our knowledge, no prior studies comparing speckle-derived strain to invasive systolic function parameters in the left ventricle in humans have been published. Greenberg et al. ${ }^{16}$ found a strong correlation between tissue Doppler-derived strain rate and catheter-derived measurements of systolic function in dogs. In their study, they found a strong correlation between peak systolic strain rate and $\mathrm{dp} / \mathrm{dt}$ for the left ventricle $(R=.78)$. In our population, using speckle tracking to calculate strain, left ventricular global longitudinal strain and strain rate correlated well with max dp/dt. Nguyen et al. compared invasive catheter measurements of left ventricular filling pressure to left ventricular longitudinal strain in fifty adults and found that there was significant correlation between the two $(R=.52, P<.001)$. We had a similar trend in our data $(R=.52, P=.08) .{ }^{7}$ This suggests that left ventricular longitudinal strain is a robust measure of ventricular function, even when DICOM, JPEG-compressed images at 30 frames per second are used for analysis. This is in agreement with findings from prior studies that have demonstrated good reproducibility of speckle tracking analysis of DICOM, compressed images for LV longitudinal strain. Risum et al. ${ }^{17}$ performed a study on speckle tracking analysis of 30 adult echocardiograms acquired using machines from two different vendors (GE and Philips). The authors found excellent intra-observer and interobserver reproducibility of LV longitudinal strain of images that have compressed frame $\operatorname{rates}(R=.98$, bias $=-.3$ and $R=.94$, bias $=0.4$, respectively) and these values were similar when acquisition frame rate images were analyzed. In a study on echocardiograms performed on 49 children, Koopman et al. ${ }^{9}$ also made similar observations. They found that longitudinal strain of the left ventricle calculated from DICOM images using vendor-independent software has reasonable agreement with minimal bias $(R=.44$, bias-1.4) when compared with longitudinal strain values from speckle tracking analysis using acquisition frame rate. 
In our study, strain rate of the left ventricle from the two-chamber view had a stronger correlation with catheter-derived max dp/dt compared to that from the four-chamber view. This finding suggests that in this patient population, the septum may not play as much of a role in contractile (systolic) function as the free walls of the left ventricle. As most of the subjects in this study were cardiac transplant recipients, a population that has a high frequency of septal dysfunction, it is unclear whether this finding is only present in this population or whether it can be generalizable to other pediatric populations. Further investigation will need to be performed to confirm this finding.

This study also demonstrates the feasibility of the speckle-derived strain measurements for both ventricles. The right ventricle was more challenging to image and analyze, but all myocardial segments tracked in $72 \%$ of the echocardiograms. The quality of left ventricle images for speckle tracking analysis was better for the four-chamber view but quite limited in the two-chamber view of the left ventricle. All myocardial segments tracked in $87.5 \%$ of all images obtained in the four-chamber view vs $56.2 \%$ of the images of the two-chamber view.

Early diastolic strain rate did not correlate with end-diastolic pressure in either ventricle in this study, which suggests that the algorithms used to calculate diastolic strain rate are not as robust as other speckle-derived function parameters. This differs from prior studies in animal and human models that demonstrated that speckle-derived peak diastolic strain rate correlates to left ventricular end-diastolic pressure. ${ }^{8,18}$ In addition, studies in the pediatric population have shown good correlation between early diastolic strain rate to end-diastolic pressure in the right ventricle. ${ }^{15}$ These studies used different vendor software to calculate diastolic strain rate, and therefore, inter-vendor differences may account for the discrepancies between the findings of our study and those of these other studies. The inconsistencies between the results of our study and prior studies may be also be related to the fact that we used compressed images with lower frame rates for speckle tracking analysis. As the peak of diastolic strain rate occurs over a short time interval, this event may be missed when speckle tracking analysis is performed with this form of echocardiographic data. This issue did not seem to affect systolic strain rate for the left ventricle as much, as we found good correlation between left ventricular systolic strain rate and max dp/dt.

\section{STUDY LIMITATIONS}

Fluid-filled catheters were used to perform invasive catheter-derived measurements which are limited by the lower natural frequency response as compared to micromanometer-tipped catheters. However, micromanometer catheter equipment was not available to the authors at the time of this study.

Echocardiograms and catheterization were not truly simultaneous. However, a large majority of echocardiographic and cardiac catheterization studies was performed under general anesthesia, albeit at slightly different moments in time. All echocardiograms were performed on the day of catheterization. As anesthetic agents and sedation can impact myocardial function and alter loading conditions, differences in echocardiographic and catheter-derived measurements may be related to differences in cardiac function between the acquisitions. 
Analysis of echocardiograms and calculation of $\mathrm{dp} / \mathrm{dt}$ was performed by a single, blinded observer and confirmed by a second blinded observer. To minimize bias, these measurements were performed at different time points, at least several days apart. Both datasets were anonymized during acquisition so that the observers were blinded.

Longitudinal strain for the right ventricle was only performed in one plane, whereas longitudinal strain for the left ventricle was performed in two views. This may impact the correlation of the right ventricular longitudinal strain with invasive catheter-based data. Future studies should evaluate the correlation of right ventricular longitudinal strain in multiple views and assess correlation of global measurements with invasive catheter-based data.

We used DICOM, JPEG-compressed images at 30 frames per second for speckle tracking analysis. Higher frame rates may increase the ability for the software to detect the movement of speckles, which can impact the analysis. In addition, JPEG compression may result in the loss of data that may be important in speckle tracking analysis, and may decrease the sensitivity of strain and strain rate detection. Speckle tracking for most major vendors is based on individual scan lines of data while the current DICOM standard only accepts pixels in a Cartesian, raster-based orientation. ${ }^{19}$ Therefore, if the software is attempting to track speckles that move in a plane outside the referenced Cartesian planes, this motion may not be tracked accurately. Our values for longitudinal strain were overall lower than previously reported values in normal healthy children with biventricular hearts ${ }^{10,11}$ but may be reasonable considering that our patients were fundamentally not "normal." For the most part, they were children who had undergone cardiac transplantation and were undergoing cardiac catheterization under anesthesia. The significance of data compression on the clinical application of speckle-derived longitudinal strain calculations is still not clear. As most echocardiography laboratories store echocardiograms according to the DICOM standard using JPEG compression at limited frame rates, the purpose of our study was to use standard clinical echocardiograms for speckle tracking analysis and compare these values to catheter-derived measurements. In light of the potential concerns regarding data compression on speckle tracking analysis, however, negative findings in this study should be taken with caution and reevaluated using uncompressed images at higher frame rates.

Some subjects had repeated measurements, and there were a limited number of catheterbased measurements in the left ventricle, which leads to an overall smaller sample size of total subjects. This may significantly limit the generalizability of our results to the general population.

\section{CONCLUSIONS}

Left ventricular global longitudinal strain and longitudinal strain rate in the left ventricular two-chamber view correlated well with systolic catheter-derived measures of function and appear to be robust measures of systolic cardiac function. Right ventricular strain did not correlate as well to catheter-derived indices of function. Our findings should be confirmed with further studies using micromanometer catheters for comparison and speckle tracking analysis on uncompressed images with higher frame rates. 


\title{
Acknowledgments
}

\author{
FUNDING
}

The authors have no financial disclosures

\section{REFERENCES}

1. Lu X, Xie M, Tomberlin D. How accurately, reproducibly, and efficiently can we measure left ventricular indices using M-mode, 2-dimensional, and 3-dimensional echocardiography in children? Am Heart J. 2008; 155:946-953. [PubMed: 18440346]

2. Amundsen BH, Helle-Valle T, Edvardsen T, et al. Noninvasive myocardial strain measurement by speckle tracking echocardiography: validation against sonomicrometry and tagged magnetic resonance imaging. J Am Coll Cardiol. 2006; 47:789-793. [PubMed: 16487846]

3. Edvardsen T, Gerber BL, Garot J, et al. Quantitative assessment of intrinsic regional myocardial deformation by Doppler strain rate echocardiography in humans: validation against threedimensional tagged magnetic resonance imaging. Circulation. 2002; 106:50-56. [PubMed: 12093769]

4. Singh GK, Cupps B, Pasque M, et al. Accuracy and reproducibility of strain by speckle tracking in pediatric subjects with normal heart and single ventricular physiology: a two-dimensional speckletracking echocardiography and magnetic resonance imaging correlative study. J Am Soc Echocardiogr. 2010; 23:1143-1152. [PubMed: 20850945]

5. Scherptong RW, Mollerna SA, Blom NA, et al. Right ventricular peak systolic longitudinal strain is a sensitive marker for right ventricular deterioration in adult patients with tetralogy of Fallot. Int $\mathrm{J}$ Cardiovasc Imaging. 2009; 25:669-676. [PubMed: 19642012]

6. Meluzin J, Spinarova L, Hude P, et al. Estimation of left ventricular filling pressures by speckle tracking echocardiography in patients with idiopathic dilated cardiomyopathy. Eur J Echocardiogr. 2011; 12:11-18. [PubMed: 20688766]

7. Nguyen JS, Lakkis NM, Bobek J, et al. Systolic and diastolic myocardial mechanics in patients with cardiac disease and preserved ejection fraction: impact of left ventricular filling pressure. J Am Soc Echocardiogr. 2010; 23:1273-1280. [PubMed: 20970305]

8. ten Brinke EA, Bertini M, Klautz RJ, et al. Noninvasive estimation of left ventricular filling pressures in patients with heart failure after surgical ventricular restoration and restrictive mitral annuloplasty. J Thorac Cardiovasc Surg. 2010; 140:807-815. [PubMed: 20117802]

9. Koopman LP, Slorach C, Manlhiot C, et al. Assessment of myocardial deformation in children using Digital Imaging and Communications in Medicine (DICOM) data and vendor independent speckle tracking software. J Am Soc Echocardiogr. 2011; 24:37-44. [PubMed: 21095099]

10. Lorch SM, Ludomirsky A, Singh GK. Maturational and growth-related changes in left ventricular longitudinal strain and strain rate measured by two-dimensional speckle tracking echocardiography in healthy pediatric population. J Am Soc Echocardiogr. 2008; 21:1207-1215. [PubMed: 18992672]

11. Marcus KA, Mavinkurve-Groothuis AM, Barends M, et al. Reference values for myocardial twodimensional strain echocardiography in a healthy pediatric and young adult cohort. J Am Soc Echocardiogr. 2011; 24:625-636. [PubMed: 21392941]

12. Levy PT, Sanchez Mejia AA, Machefsky A, et al. Normal ranges of right ventricular systolic and diastolic strain measures in children: a systematic review and meta-analysis. J Am Soc Echocardiogr. 2014; 27:549-560. [PubMed: 24582163]

13. Schlangen J, Petko C, Hansen JH, et al. Two-dimensional global longitudinal strain rate is a preload independent index of systemic right ventricular contractility in hypoplastic left heart syndrome patients after Fontan operation. Circ Cardiovasc Imaging. 2014; 7:880-886. [PubMed: 25270741]

14. Husain N, Gokhale J, Nicholson L, et al. Comparing echocardiographic assessment of systolic function with catheterization data in patients with single right ventricles. Acta Cardiol. 2014; 69:281-288. [PubMed: 25029873] 
15. Husain N, Gokhale J, Nicholson L, et al. Noninvasive estimation of ventricular filling pressures in patients with single right ventricles. J Am Soc Echocardiogr. 2013; 26:1330-1336. [PubMed: 24035700]

16. Greenberg NL, Firstenberg MS, Castro PL, et al. Doppler-derived myocardial systolic strain rate is a strong index of left ventricular contractility. Circulation. 2002; 105:99-105. [PubMed: 11772883]

17. Risum N, Ali S, Olsen NT, et al. Variability of global left ventricular deformation analysis using vendor dependent and independent two-dimensional speckle-tracking software in adults. J Am Soc Echocardiogr. 2012; 25:1195-1203. [PubMed: 22981228]

18. Wang J, Khoury DS, Thohan V, et al. Global diastolic strain rate for the assessment of left ventricular relaxation and filling pressures. Circulation. 2007; 115:1376-1383. [PubMed: 17339549]

19. Kaul S, Miller JG, Grayburn PA, et al. A suggested roadmap for cardiovascular ultrasound research for the future. J Am Soc Echocardiogr. 2011; 24:455-464. [PubMed: 21440216] 

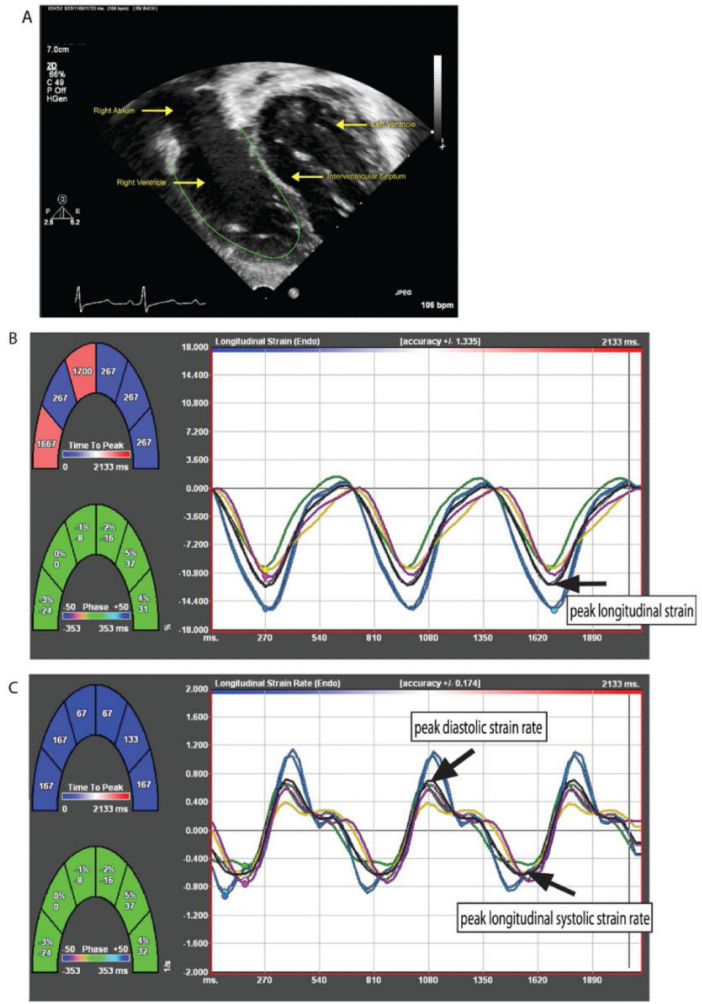

FIGURE 1.

Images of a right ventricular four-chamber view on Tomtec 2D Cardiac Performance Analysis software for speckle tracking analysis. A. Echo image with user-defined region of interest just beneath the endocardium. This border is visually inspected for tracking throughout the cardiac cycle. B. Longitudinal strain curve of all segments in three cardiac cycles. The black line represents the average of all segments, and its nadir was used as the global longitudinal strain, as noted. C. Longitudinal strain rate curve of all segments in three cardiac cycles. The black line represents the average strain rate curve of all segments, and its nadir and peak were used as the peak longitudinal systolic strain rate and diastolic strain rate, as illustrated 

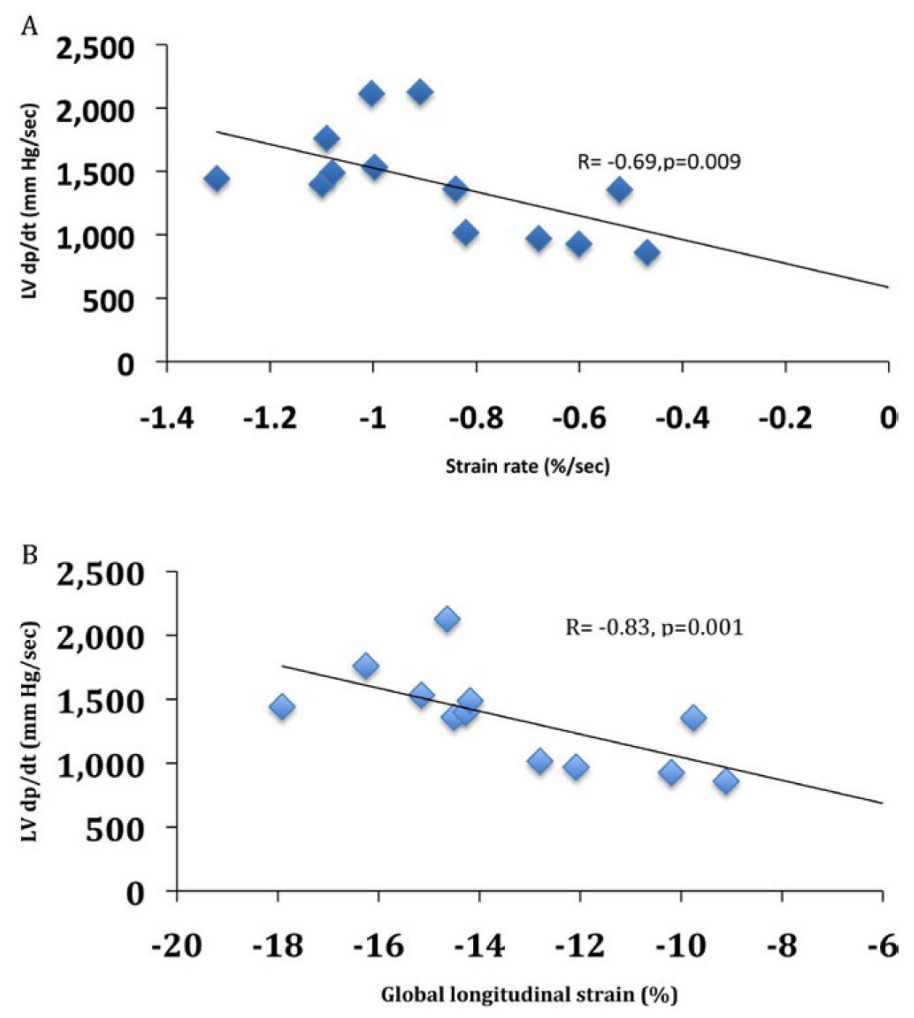

FIGURE 2.

Correlation plots of LV dp/dt with LV two-chamber longitudinal strain rate (A) and global longitudinal strain (B) 

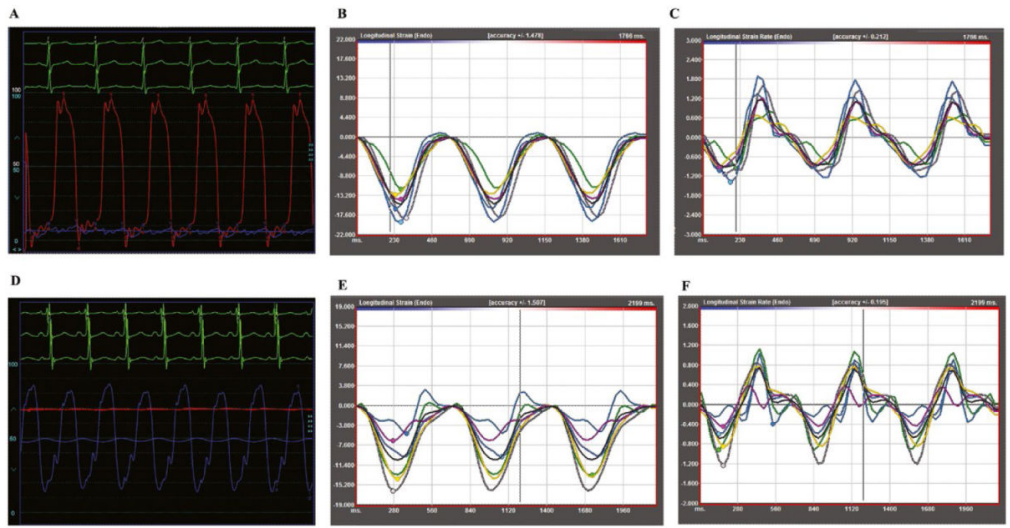

FIGURE 3.

Catheter pressure tracings and longitudinal strain and strain rate curves of the left ventricle of a subject with normal LV function (A-C) and a subject with poor LV function (D-E). (A) catheter-derived LV pressure tracing in a subject with good LV function who is postcardiac transplant (B) LV longitudinal strain curve and (C) LV longitudinal strain rate curve for this same patient using speckle tracking analysis of the echocardiographic images for this same patient (D) catheter LV pressure tracing in a subject with dilated cardiomyopathy and poor LV function (E) LV longitudinal strain curve and (F) LV longitudinal strain rate curve for this same patient using speckle tracking analysis on the LV echocardiographic images 


\section{TABLE 1}

Hemodynamic and echocardiographic variables

\begin{tabular}{|c|c|c|}
\hline & Median & Range \\
\hline Age (y) & 4 & 0.3 to 20 \\
\hline Weight (kg) & 15.7 & 6 to 72.8 \\
\hline BSA & 0.64 & 0.28 to 1.88 \\
\hline Heart rate & 105 & 57 to 168 \\
\hline $\mathrm{RV}^{a}$ end-diastolic pressure & 6 & 2 to 22 \\
\hline $\mathrm{RV}^{a} \mathrm{dp} / \mathrm{dt}$ & 344 & 99 to 748 \\
\hline Cardiac Index & 3.37 & 1.84 to 4.92 \\
\hline $\operatorname{PCWP}^{b}$ & 10 & 6 to 32 \\
\hline $\mathrm{LV}^{\mathcal{c}}$ end-diastolic pressure & 11 & 8 to 17 \\
\hline $\mathrm{LV}^{\mathcal{c}} \mathrm{dp} / \mathrm{dt}$ & 1378 & 726 to 2127 \\
\hline $\mathrm{RV}^{a}$ longitudinal strain & -14.7 & -3.7 to -21 \\
\hline $\mathrm{RV}^{a}$ longitudinal strain rate & -0.96 & -0.53 to -1.65 \\
\hline $\mathrm{RV}^{a}$ diastolic strain rate & 1.05 & 0.5 to 1.81 \\
\hline $\mathrm{RV}^{a}$ ejecton fraction & 44.5 & 12 to 69 \\
\hline $\mathrm{LV}^{c}$ longitudinal strain four-chamber view & -13.8 & -2.4 to -19.7 \\
\hline $\mathrm{LV}^{c}$ longitudinal strain rate four-chamber view & -0.95 & -0.27 to -1.45 \\
\hline $\mathrm{LV}^{c}$ diastolic strain rate four-chamber view & 1.09 & 0.27 to 1.82 \\
\hline $\mathrm{LV}^{c}$ longitudinal strain two-chamber view & -13.98 & -2.73 to -22.37 \\
\hline $\mathrm{LV}^{c}$ longitudinal strain rate two-chamber view & -0.96 & -0.28 to -1.63 \\
\hline $\mathrm{LV}^{\mathcal{c}}$ ejecton fraction four-chamber view & 48 & 11 to 62 \\
\hline $\mathrm{LV}^{c}$ ejecton fraction two-chamber view & 47 & 6 to 64 \\
\hline Global $\mathrm{LV}^{c}$ longitudinal strain & -13.82 & -2.65 to -18.57 \\
\hline Global $\mathrm{LV}^{\mathcal{c}}$ longitudinal strain rate & -0.96 & -0.27 to -1.38 \\
\hline Global $\mathrm{LV}^{\mathcal{c}}$ diastolic strain rate & 1.09 & 0.28 to 1.49 \\
\hline
\end{tabular}

${ }^{a} \mathrm{RV}$, right ventricle;

$b_{\text {PCWP, pulmonary capillary wedge pressure; }}$

$c_{\mathrm{LV}}$, left ventricle 


\section{TABLE 2}

Image quality

\begin{tabular}{llll}
\hline & Grade 1 & Grade 2 & Grade 3 \\
\hline $\mathrm{RV}^{a}$ four-chamber & $72 \%$ & $16 \%$ & $12 \%$ \\
\hline $\mathrm{LV}^{b}$ four-chamber & $87.5 \%$ & $6.25 \%$ & $6.25 \%$ \\
\hline $\mathrm{LV}^{b}$ two-chamber & $56.25 \%$ & $21.13 \%$ & $12.5 \%$ \\
\hline${ }^{a} \mathrm{RV}$, right ventricle & & & \\
$b_{\mathrm{LV}}$, left ventricle & & & \\
\end{tabular}


TABLE 3

Correlation between catheter-derived and speckle-derived cardiac function measurements

\begin{tabular}{|c|c|c|c|c|c|c|}
\hline & \multicolumn{2}{|l|}{$\mathrm{RV}^{a} \mathrm{EDP}^{b}$} & \multicolumn{2}{|l|}{$\mathrm{RV}^{a} \mathrm{dp} / \mathrm{dt}$} & \multicolumn{2}{|c|}{ Cardiac Index } \\
\hline & $\begin{array}{l}\text { Correlation } \\
\text { coefficient }\end{array}$ & $P$ - value & $\begin{array}{l}\text { Correlation } \\
\text { coefficient }\end{array}$ & $P$-value & $\begin{array}{l}\text { Correlation } \\
\text { coefficient }\end{array}$ & $P$-value \\
\hline $\mathrm{RV}^{a}$ longitudinal strain & .14 & .38 & .36 & .02 & -.28 & .07 \\
\hline $\mathrm{RV}^{a}$ diastolic strain rate & .307 & .04 & N/A & N/A & -.67 & .66 \\
\hline \multirow[t]{3}{*}{$\mathrm{RV}^{a}$ ejecton fracton } & .107 & .49 & -.16 & .32 & .09 & .57 \\
\hline & \multicolumn{2}{|l|}{$\mathbf{L V}^{b} \operatorname{EDP}^{c}$} & \multicolumn{2}{|l|}{$L V^{b} \mathrm{dp} / \mathrm{dt}$} & \multicolumn{2}{|l|}{ Cardiac index } \\
\hline & $\begin{array}{l}\text { Correlation } \\
\text { coefficient }\end{array}$ & $P$-value & $\begin{array}{l}\text { Correlation } \\
\text { coefficient }\end{array}$ & $P$-value & $\begin{array}{l}\text { Correlation } \\
\text { coefficient }\end{array}$ & $P$-value \\
\hline $\mathrm{LV}^{b}$ global longitudinal strain & .52 & .08 & -.83 & .001 & .038 & .399 \\
\hline LV two-chamber longitudinal strain & .40 & .18 & -.49 & .09 & -.14 & .37 \\
\hline LV four-chamber longitudinal strain & .63 & .01 & -.4 & .14 & -.24 & .1 \\
\hline $\mathrm{LV}^{b}$ two-chamber longitudinal strain rate & .64 & .02 & -.69 & .01 & -.2 & .27 \\
\hline $\mathrm{LV}^{b}$ global diastolic strain rate & -.32 & .34 & N/A & N/A & .17 & .37 \\
\hline
\end{tabular}

${ }^{a} \mathrm{RV}$, right ventricle;

$b_{\mathrm{LV}}$, left ventricle;

${ }^{c}$ EDP, end-diastolic pressure 


\section{TABLE 4}

Correlation between catheter-derived and speckle-derived cardiac function measurements for Grade 1 quality images only

\begin{tabular}{|c|c|c|c|c|c|}
\hline & \multirow[b]{2}{*}{$\mathbf{N}$} & \multicolumn{2}{|l|}{$\mathbf{R V}^{a} \operatorname{EDP}^{c}$} & \multicolumn{2}{|l|}{$\mathrm{RV}^{a} \mathrm{dp} / \mathrm{dt}$} \\
\hline & & Correlaton coefficient & $P$-value & Correlation coefficient & $P$-value \\
\hline $\mathrm{RV}^{a}$ longitudinal strain & 36 & .001 & .96 & .02 & .2 \\
\hline $\mathrm{RV}^{a}$ diastolic strain rate & 36 & .05 & .76 & N/A & N/A \\
\hline \multirow[t]{3}{*}{$\mathrm{RV}^{a}$ ejection fracton } & 36 & .18 & .3 & -.08 & .6 \\
\hline & \multirow[b]{2}{*}{$\mathrm{N}$} & \multicolumn{2}{|l|}{$\mathbf{L V}^{b} \mathrm{EDP}^{c}$} & \multicolumn{2}{|l|}{$L V^{b} \mathrm{dp} / \mathrm{dt}$} \\
\hline & & Correlation coefficient & $P$-value & Correlation coefficient & $P$-value \\
\hline $\mathrm{LV}^{b}$ global longitudinal strain & 9 & .82 & .09 & -.9 & .04 \\
\hline $\mathrm{LV}^{b}$ global diastolic strain rate & 9 & -.42 & .49 & N/A & N/A \\
\hline $\begin{array}{l}{ }_{\mathrm{RV}} \text {, right ventricle; } \\
\mathrm{LV} \text {, left ventricle; }\end{array}$ & & & & & \\
\hline
\end{tabular}


TABLE 5

Inter-observer reproducibility of speckle-derived strain measurements

\begin{tabular}{llll}
\hline & $\begin{array}{l}\text { Longitudinal } \\
\text { strain (ICC) }\end{array}$ & $\begin{array}{l}\text { Longitudinal } \\
\text { strain rate (ICC) }\end{array}$ & $\begin{array}{l}\text { Early diastolic } \\
\text { strain rate (ICC) }\end{array}$ \\
\hline $\begin{array}{l}\mathrm{LV}^{a} \\
\text { four-chamber }\end{array}$ & .76 & .71 & .55 \\
\hline $\begin{array}{l}\mathrm{LV}^{a} \\
\text { two-chamber }\end{array}$ & .78 & .67 & .41 \\
\hline $\mathrm{Global} \mathrm{LV}$ & .8 & .71 & .6 \\
\hline $\mathrm{RV}^{b}$ & .88 & .77 & .71 \\
\hline
\end{tabular}

${ }^{a} \mathrm{LV}$, left ventricle;

$b_{\mathrm{RV} \text {, right ventricle }}$ 